Case Report

\title{
Pulmonary Histiocytic Sarcoma: A Case Report and Literature Review
}

\author{
Veljko Flego 1, *, Helmut Popper², Darian Volaric ${ }^{3}$, Ljiljana Bulat-Kardum ${ }^{1}$ \\ ${ }^{1}$ Department of Pulmonology, Clinical Hospital Centre Rijeka, Rijeka, Croatia \\ ${ }^{2}$ Institute of Pathology, Medical University of Graz, Graz, Austria \\ ${ }^{3}$ Primary Health Care Office, Health Centre of Primorsko-Goranska County, Rijeka, Croatia
}

\section{Email address:}

veljko.flego@medri.uniri.hr (V. Flego), helmut.popper@medunigraz.at (H. Popper),darian.volaric7@gmail.com (D. Volaric), bulat.ljiljana@medri.uniri.hr (L. Bulat-Kardum)

${ }^{*}$ Corresponding author

\section{To cite this article:}

Veljko Flego, Helmut Popper, Darian Volaric, Ljiljana Bulat-Kardum. Pulmonary Histiocytic Sarcoma: A Case Report and Literature Review. American Journal of Internal Medicine. Vol. 5, No. 5, 2017, pp. 91-94. doi: 10.11648/j.ajim.20170505.16

Received: May 14, 2017; Accepted: June 6, 2017; Published: October 24, 2017

\begin{abstract}
Pulmonary histiocytic sarcoma is a rare, but highly malignant disease. Its low incidence imposes significant difficulties on physicians confronted with affected patients. The reported patient is a 24-year old male with histiocytic sarcoma of the lung. Left lower lobectomy was performed. Histologically, tumor cells had no features of carcinoma. Several entities were raised as differentials: large $\mathrm{B}$ or $\mathrm{T}$ cell lymphomas, metastatic melanoma, sarcoma, undifferentiated carcinoma, NK cell lymphoma. Immunohistochemically, melanoma, carcinoma, undifferentiated epithelioid sarcoma and also different types of lymphoma were excluded. Therefore, tumors of the histiocytic and dendritic cell lineage had to be considered. Tumors of the dendritic cell lineage were also immunohistochemically excluded, leaving histiocytic sarcoma by exclusion. The patient was followed for six years and six months, with no signs of recurrence of the tumor. Histiocytic sarcomas are tumors of uncertain behavior, with some progressing quickly, and others having a much slower course. As these tumors are rare, there is not much information, although a low number of mitosis might point to a less aggressive course. In the present patient with an unifocal disease, surgical excision was sufficient, without adjuvant radiotherapy and chemotherapy.
\end{abstract}

Keywords: Neoplasms, Histiocytic Sarcoma, Lung, Immunohistochemistry, Surgical Treatment

\section{Introduction}

Histiocytic sarcoma (HS) is a rare hematological malignant neoplasm composed of cells showing morphologic and immunophenotypic evidence of histiocytic differentiation [1]. HS usually affects the lymph nodes, skin, liver, spleen, soft tissue, and the gastrointestinal tract, whereas HS arising in the lung has very rarely been reported [2]. Reported cases of HS located in the central nervous system (CNS) and bone marrow [3-5] are regarded as aggressive hematopoietic neoplasm with a strong potential for systemic spread and a poor response to therapy [6].

\section{Case Report}

The reported patient is a 24-year old male smoker, until then healthy, who reported subfebrile temperatures for two months. Physical examination was normal. Erythrocyte sedimentation rate (ESR) was $110 \mathrm{~mm} / \mathrm{h}$ (reference interval: $2-13 \mathrm{~mm} / \mathrm{h}$ ), a complete blood count showed a leukocyte (WBC) count of 8.9 x109/L (reference interval: 4.0-11.0 x109/L), with normal percentage of particular classes of leukocytes. The hemoglobin was $111 \mathrm{~g} / \mathrm{L}$ (reference interval: $138-175 \mathrm{~g} / \mathrm{L}$ ), and the platelet count was $607 \times 109 / \mathrm{L}$ (reference interval: $158-424$ x109/L). C-reactive protein (CRP) was $158.1 \mathrm{mg} / \mathrm{L}$ (reference interval: $<5.0 \mathrm{mg} / \mathrm{L}$ ). Serum biochemistry revealed an elevated alkaline phosphatase (ALP) level of $248 \mathrm{U} / \mathrm{L}$ (reference interval: 60-142 U/L), and gamma glutamyl transferase (GGT) level of $95 \mathrm{U} / \mathrm{L}$ (reference interval: 11-55 $\mathrm{U} / \mathrm{L}$ ). The aspartate aminotransferase (AST), alanine aminotransferase (ALT), lactate dehydrogenase (LD), and 
total bilirubin level were normal. Tumor markers [CA (cancer antigen) 19-9, CA (cancer antigen) 125, CEA (carcinoembryonic antigen), PSA (prostate specific antigen), AFP (alpha-1-phetoprotein), NSE (neuron-specific enolase), CYFRA (cytokeratin 19 fragments) 21-1, B2M (beta-2 microglobulin)] in the serum were normal.

Chest radiography revealed a mass shadow measuring 70 $\mathrm{mm}$ in diameter in the left lower lung lobe. Multi slice computed tomography (MSCT) of the chest showed in the central part of the left 9th segment of lung, retrocardially, a well-defined, lobulated solid tumor mass, approximately $60 \mathrm{x}$ $38 \times 70 \mathrm{~mm}$ in size. The tumor completely obstructed the B9 segmental bronchus (Figure 1). No mediastinal lymph node metastasis or other organ metastases were observed. Bronchoscopy revealed a necrotic tumor obstructing the entry of the left B9 bronchus (Figure 2). Histological and cytological findings of tumor forceps and brush biopsy were not able to establish the diagnosis. The patient underwent open lung biopsy, left lower lobe resection and mediastinal lymphadenectomy.
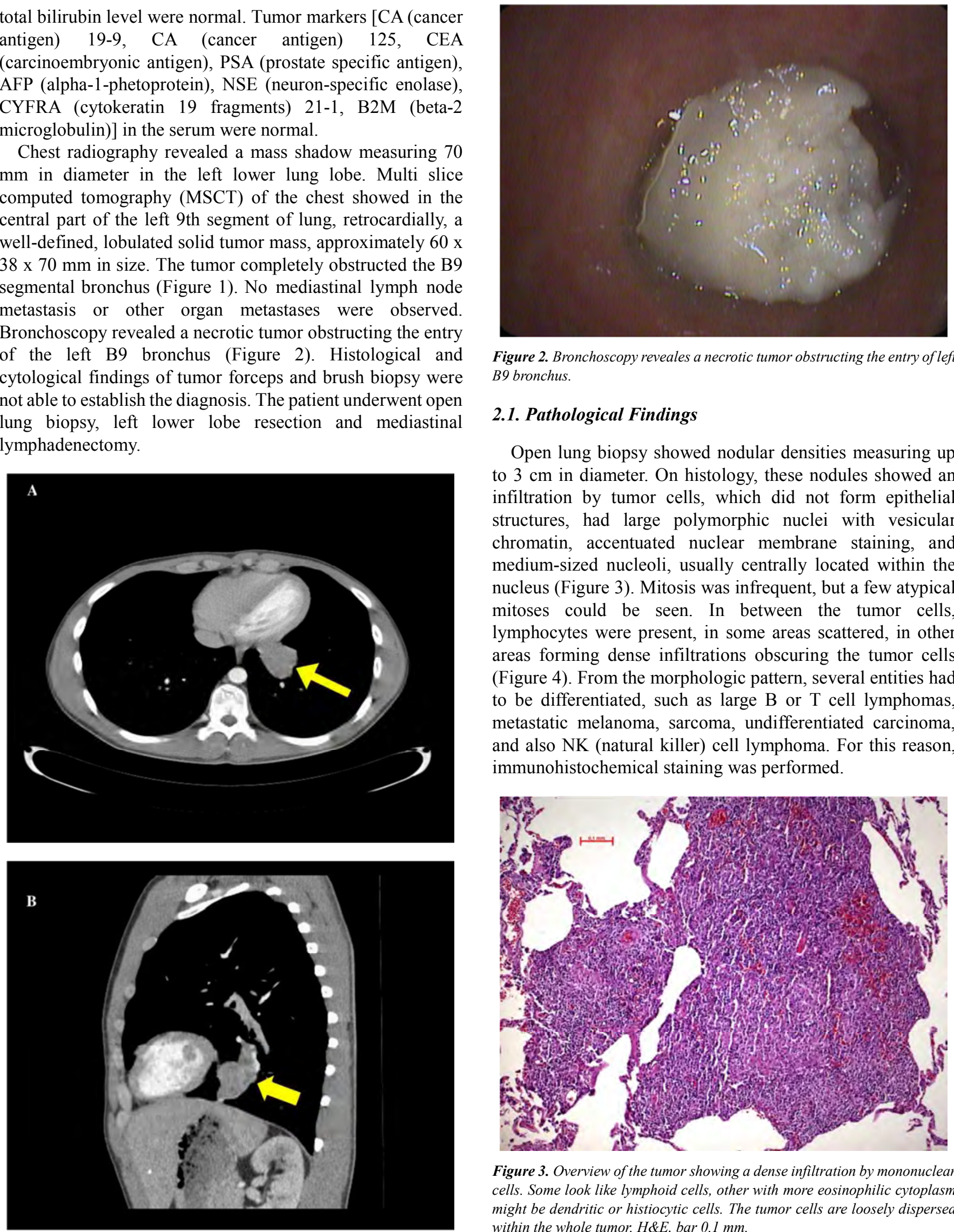

Figure 2. Bronchoscopy reveales a necrotic tumor obstructing the entry of left B9 bronchus.

\subsection{Pathological Findings}

Open lung biopsy showed nodular densities measuring up to $3 \mathrm{~cm}$ in diameter. On histology, these nodules showed an infiltration by tumor cells, which did not form epithelial structures, had large polymorphic nuclei with vesicular chromatin, accentuated nuclear membrane staining, and medium-sized nucleoli, usually centrally located within the nucleus (Figure 3). Mitosis was infrequent, but a few atypical mitoses could be seen. In between the tumor cells, lymphocytes were present, in some areas scattered, in other areas forming dense infiltrations obscuring the tumor cells (Figure 4). From the morphologic pattern, several entities had to be differentiated, such as large B or $\mathrm{T}$ cell lymphomas, metastatic melanoma, sarcoma, undifferentiated carcinoma, and also NK (natural killer) cell lymphoma. For this reason, immunohistochemical staining was performed.

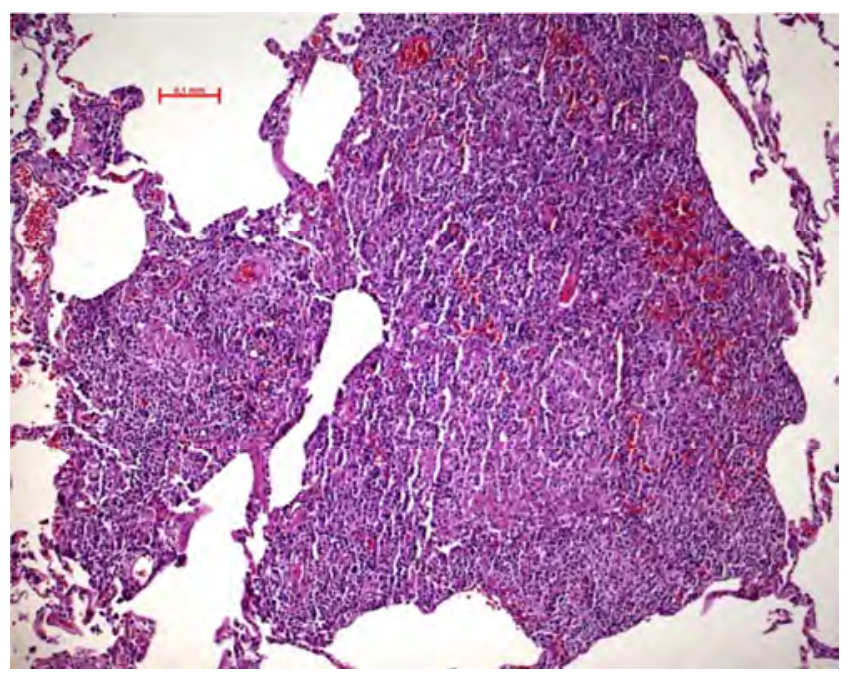

Figure 3. Overview of the tumor showing a dense infiltration by mononuclear cells. Some look like lymphoid cells, other with more eosinophilic cytoplasm might be dendritic or histiocytic cells. The tumor cells are loosely dispersed within the whole tumor. $H \& E$, bar $0.1 \mathrm{~mm}$.

Figure 1. Thoracic axial (A) and sagittal (B) MSCT scan showing a left lobulated solid tumor (arrows). 


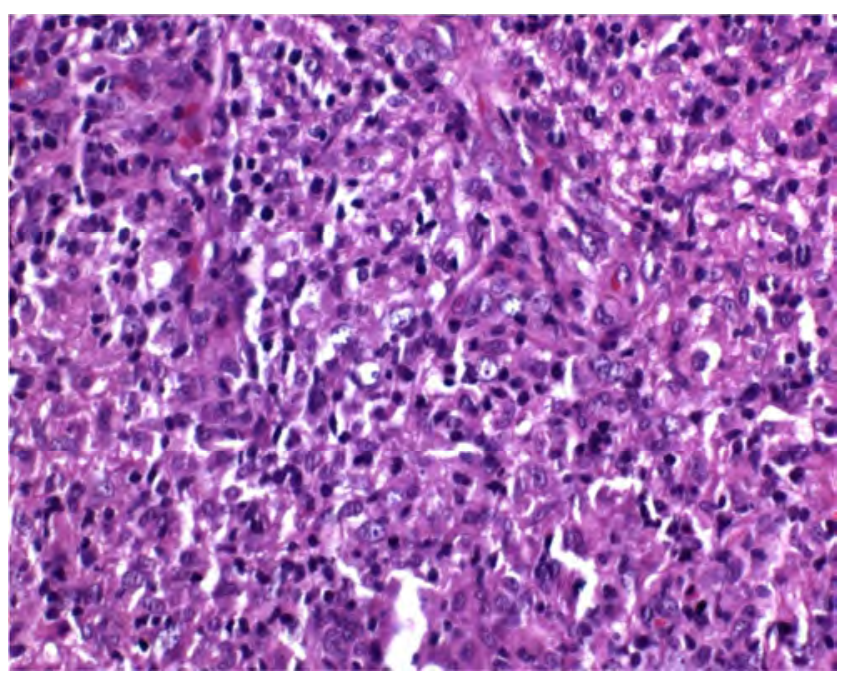

Figure 4. High power magnification of the tumor. There are large tumor cells embedded in a lymphocytic background infiltration. The nuclei of the tumor cells are large, have medium-sized nucleoli and a vesicular chromatin. The cytoplasm is eosinophilic. $H \& E$, $x 400$.

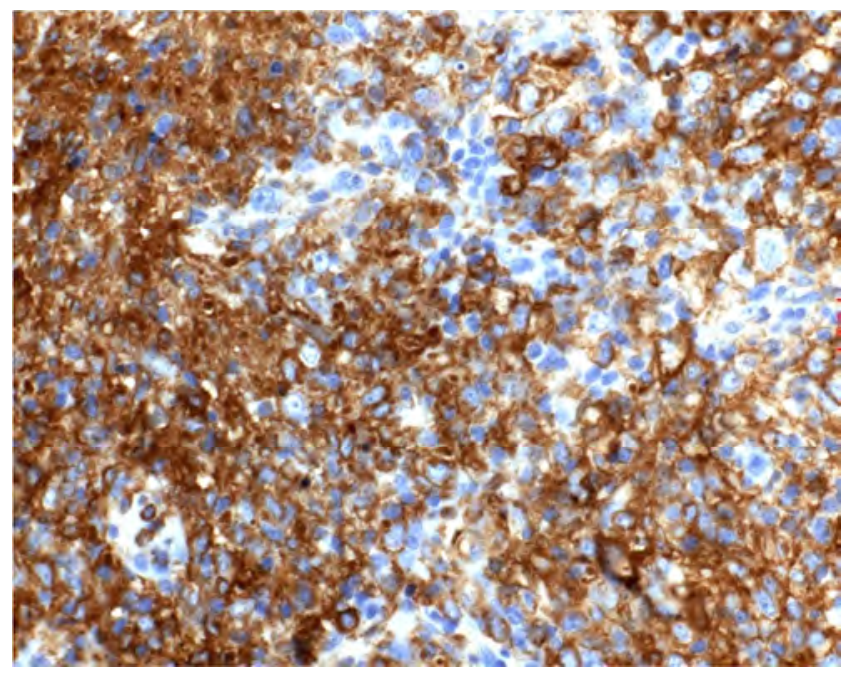

Figure 5. Immunohistochemistry for CD14 shows positively stained tumor cells, typically membraneous. Magnification $x 400$.

\subsection{Immunohistochemistry}

The tumor cells were positive for CD14 (Figure 5), HLA-DR, CD68, lysozyme, and focally some cells also stained for CD35. The tumor cells were negative for pan-cytokeratin, NCAM, S100 protein, EMA, HMB45, podoplanin, inhibin, CD99, CD117, desmin. Therefore melanoma, carcinoma, undifferentiated epithelioid sarcoma and also different types of lymphoma could be sorted out. With the positive stains, tumors of the histiocytic and dendritic cell lineage had to be considered. Due to the negative staining for S100 protein and the positivity for dendritic/histiocytic cell markers, a diagnosis of histiocytic sarcoma/tumor was rendered. Dendritic cell sarcomas should be either positive for CD35 (follicular) or CD83 (interdigitating) or usually at least focally positive for $\mathrm{S} 100$ protein and thus tumors of the dendritic cell lineage could also be excluded, leaving histiocytic tumors by exclusion. The patient has been followed up on a three- and six-month basis, including clinical examinations, positron emission tomography/computed tomography (PET/CT) and CT scans. The postoperative course has been favorable for six years and six months, without additional treatment. Consent, for the publication for this case report and any additional related information was taken from the patient involved in the study.

\section{Discussion}

Histiocytic sarcoma is a malignant neoplasm recognized by the expression of histiocytic markers. HS is rare; the median age of a patient at the time of diagnosis is usually 55 years [2]. There is no obvious sex predilection. Histologically, the tumor cells are large, round to oval, have rich eosinophilic cytoplasm and are consequently similar to epithelioid histiocytes. These neoplasms usually have large, vesicular nuclei, prominent nucleoli and cytoplasmic vacuoles. Also common are giant multinucleated cells and a conspicuous inflammatory surrounding including plasma cells, lymphocytes, neutrophils, and eosinophils. Mitoses are variably present in HS.

Diagnosis is based on immunophenotypic researches. The neoplasm cells are positive for histiocyte-linked markers such as lysozyme, CD163 and CD68. Histiocytes are likewise frequently positive for HLA-DR, CD45RO and CD45, and can express $\mathrm{CD} 15$ and $\mathrm{CD} 4$. The proliferation pace as determined by $\mathrm{Ki}-67$ is immensely variable in HS studies, with a mean of $25 \%$ [7]. Neoplasm cells are negative for markers of more specialized histiocytes and thus negative for markers linked with interdigitating or follicular dendritic cell markers (CD83, CD35, CD23, CD21) or Langerhans cells (Langerin/CD207 and CD1a). In HS studies, S100 may be expressed, but mostly focally or less intensely than in interdigitating dendritic cell sarcoma. Likewise, myeloid-related cell markers (CD13, CD33) and myeloperoxidase are usually not expressed by the cells of HS. Pan T- or B-cell markers are not expressed by the cells of HS.

HS is treatment-resistant, the prognosis is poor, many cases are progressive, and $60-80 \%$ of cases exhibit tumor progression [1]. The prognosis of local small lesions is favorable [2]. The tumor size of the present patient was larger compared with reported cases of HS originating in the lung. It was localized in the lung, completely surgically removed, showing the longest follow up of pulmonary HS so far. Only three cases of HS originating in the lung have previously been reported $[2,6,7]$. In Table 1 are summarized the findings of the three previous published cases as well as the present case. Pulmonary HS can also be a part of multi-organic disease [8].

There is no standardized treatment for patients with HS. For patients with localized disease, the choice of treatment is surgical excision with adjuvant radiotherapy, depending on the location and extent of the disease. For patients with nonresectable, multifocal or aggressive disease, chemotherapy with several chemotherapeutic agents simultaneously is taken into account [9]. Target therapy (imatinib, sorafenib, bevacizumab, alemtuzumab) gives encouraging results $[10,11]$. 
Table 1. Pulmonary histiocytic sarcoma.

\begin{tabular}{lllllll}
\hline Case No. & Authors & Published year & Age & Sex & Tumor location & Tumor size \\
\hline 1 & Hornick, et al. & 2004 & 63 & M & Right lung lower lobe & $68 \mathrm{~mm}$ \\
2 & Stacher, et al. & 2009 & 23 & M & Right lung & $4 \mathrm{~mm}$ \\
3 & Tomita, et al. & 2015 & 16 & M & Right lung (S6) & $32 \mathrm{~mm}$ \\
4 & Current study & & 24 & M & Left lung (S9) & $70 \mathrm{~mm}$ \\
\hline
\end{tabular}

Table 1. Continued.

\begin{tabular}{lllll}
\hline Case No. & Authors & Histology & Therapy & Outcome \\
\hline 1 & Hornick, et al. & NA & Chemo & NA \\
2 & Stacher, et al. & Histiocyte-like cells with moderate pleomorphism & Surgical resection & No recurrence within 1 year \\
3 & Tomita, et al. & Spindle cells and foam cells & Surgical resection & No recurrence within 2 years \\
4 & Current study & Single cells with large, polymorphic, vesicular nuclei & Surgical resection & No recurrence within 6.5 years \\
\hline
\end{tabular}

M, male; NA, not available; Chemo, chemotherapy.

\section{Conclusion}

In conclusion, histiocytic sarcomas are tumors of uncertain behavior, with some progressing quickly, and others having a much slower course. As these tumors are rare, there is not much information, although a low number of mitosis might point to a less aggressive course. In the present patient with an unifocal disease, surgical excision was sufficient, without adjuvant radiotherapy and chemotherapy.

\section{References}

[1] Takahashi E, Nakamura S. Histiocytic sarcoma: an updated literature review based on the 2008 WHO classification. J Clin Exp Hematop 2013; 53 (1): 1-8.

[2] Hornick JL, Jaffe ES, Fletcher CD. Extranodal histiocytic sarcoma: clinicopathologic analysis of 14 cases of a rare epithelioid malignancy. Am J Surg Pathol 2004; 28 (9): 1133-44.

[3] Chen CJ, Williams EA, Mc Aneney TE, Williams BJ, Mandell JW, Shaffrey ME. Histiocytic sarcoma of the cavernous sinus: case report and literature review. Brain Tumor Pathol 2015; 32 (1): 66-71.

[4] Sun W, Nordberg ML, Fowler MR. Histiocytic sarcoma involving the central nervous system: clinical, immunohistochemical, and molecular genetic studies of a case with review of the literature. Am J Surg Pathol 2003 Feb; 27 (2): $258-65$

[5] Jang YH, Park CJ, Huh JR, Jang S, Chi HS. A case of histiocytic sarcoma diagnosed by bone marrow biopsy in a patient suffering from fever for 8 months. Korean J Lab Med 2009; 29 (4): 282-5.

[6] Stacher E, Beham-Schmid C, Terpe HJ, Simiantonaki N, Popper HH. Pulmonary histiocytic sarcoma mimicking pulmonary Langerhans cell histiocytosis in a young adult presenting with spontaneous pneumothorax: a potential diagnostic pitfall. Virchows Arch 2009; 455: 187-90.

[7] Tomita S, Ogura $\mathrm{G}$, Inomoto $\mathrm{C}$, et al. Histiocytic sarcoma originating in the lung in a 16-year-old male. J Clin Exp Hematop 2015; 55 (1): 45-9.

[8] Aichaouia C, Daboussi S, Haddaoui A, et al. Unusual lung localization of histiocytic sarcoma. Rev Pneumol Clin 2012; 68 (5): 318-22.

[9] Buonocore S, Valente AL, Nightingale D, Bogart J, Souid AK. Histiocytic sarcoma in a 3-year-old male: a case report. Pediatrics 2005; 116 (2): e322-5.

[10] Schlick K, Aigelsreiter A, Pichler M, et al. Histiocytic sarcoma - targeted therapy: novel therapeutic options? A series of 4 cases. Onkologie 2012; 35 (7-8): 447-50.

[11] Shukla N, Kobos R, Renaud T, et al. Successful treatment of refractory metastatic histiocytic sarcoma with alemtuzumab. Cancer 2012; 118 (15): 3719-24. 